\title{
Synbiotics, Surgical Infection and Colonization Resistance
}

Shunichiro Komatsu*

Department of Gastroenterological Surgery, Aichi Medical University, Nagakute, Aichi, Japan

*Corresponding author: Shunichiro Komatsu, MD, Department of Gastroenterological Surgery, Aichi Medical University, 1-1, Yazakokarimata, Nagakute, Aichi 480-1195, Japan, Tel: +81-561-62-3311; Fax: +81-561-63-3208; E-mail: skomat2718@gmail.com

Received date: February 17, 2016; Accepted date: April 08, 2016; Published date: April 15, 2016

Copyright: (C) 2016 Komatsu S. This is an open-access article distributed under the terms of the Creative Commons Attribution License, which permits unrestricted use, distribution, and reproduction in any medium, provided the original author and source are credited.

\begin{abstract}
In our recent randomized controlled study to assess the effect of perioperative oral administration of synbiotics on surgical outcome in patients undergoing laparoscopic colorectal resection, the efficacy of synbiotics as a treatment to reduce the incidence of infectious complications was not validated. However, this study incorporated molecularbased methods and a large sample size, and microbiological examinations showed that dysbiosis induced by surgery was greatly improved by perioperative synbiotic treatment, resulting in the decrease of potentially pathogenic bacteria, such as Clostridium difficile. This study also provided novel evidence that could explain, in part, the mechanisms whereby probiotics enhance colonization resistance in the gastrointestinal tract.
\end{abstract}

Keywords: Postoperative complication; Probiotics; Bifidobacterium; Clostridium difficile; Bacterial translocation; Short chain fatty acids

\section{Description}

The gut microbiota performs a wide range of beneficial functions for human health and homeostasis, maintaining nutrition, strengthening the immune system and improving colonization resistance. There is also increasing evidence that gut microbial imbalances (dysbiosis) are associated with various intestinal-related diseases such as inflammatory bowel diseases and irritable bowel syndrome, as well as infectious complications after surgery [1-4].

Probiotics are defined as live microorganisms that have positive effects on human health when ingested in sufficient amounts. The therapeutic effects of probiotic administration on disorders presumably associated with dysbiosis have been extensively examined [1-3,5-7]. Several randomized controlled trials, including pancreatoduodenectomy, hepatobiliary resection and liver transplantation, demonstrate that the use of probiotics in patients undergoing abdominal surgery is a promising approach to the prevention of post-operative infectious complications [8-11]. Nevertheless, the effectiveness of probiotics treatment for prevention of infectious complications after colorectal surgery remains inconclusive $[1,3,12-21]$, possibly due to differences in patient groups, varying preparations of probiotics, and heterogeneity in the quality of methods. Whether or not beneficial bacteria actually survive in the intestine also remains unclear.

We recently published the results of a randomized controlled study to assess the effect of perioperative oral administration of synbiotics, a combination of probiotics (Lactobacillus casei strain Shirota and Bifidobacterium breve strain Yakult) and prebiotics (dietary supplement of probiotics), on surgical outcome in patients undergoing laparoscopic colorectal resection [22]. In this study, surgical site infection (SSI) occurred in $17.3 \%$ of synbiotics group patients and in $22.7 \%$ of control group patients (OR $0.761,95 \%$ CI $050-1.16 ; \mathrm{p}=0.20$ ). Overall, the rate of postoperative complications, including anastomotic leakage, did not differ significantly between groups. The efficacy of perioperatively administered synbiotics was not validated as a treatment to reduce the incidence of infectious complications after surgery. In previous studies of hepatobiliary, pancreatic, and esophageal surgery, usefulness of synbiotics/probiotics as prophylaxis has been described in the context of bacterial translocation (BT), associated with an altered intestinal barrier and immune function [8-11,23-25]. The conditions for use of prophylactic agents in the colon and rectum may, however, be different. In these organs BT plays a less prominent role and the potential risk of intraoperative contamination appears far greater.

In contrast to our results, some recent randomized controlled studies showed a considerable reduction of infectious complications after open radical colorectal surgery with a combined probiotic formula over placebo $[15,21,26]$. It is not likely that the inconsistent results may attribute to the difference in administered probiotics, because our formula was shown to distinguishingly improve microbial imbalance as well as reduction in organic acids induced by surgery. Rather, the colorectal resections with open laparotomy may have elicited greater surgical stress or systemic inflammatory response, in which effects of probiotics protecting against BT could emerge more prominently, compared with our laparoscopic surgery.

In the same series of patients, microbiological examinations using molecular-based methods with a large sample size provided novel and precise evidence that significant changes in fecal bacterial composition occur following laparoscopic colorectal resection [22]. Such microbial imbalance induced by mechanical bowel preparation [27], surgical stress or manipulation of the intestine was improved by perioperative synbiotic treatment whereby decreases of potentially pathogenic bacteria, such as Clostridium difficile (CD) (4\% in synbiotics group vs. $13 \%$ in control group), and increases in beneficial bacteria were observed. These results also indicate that the administration of synbiotics restores the diminished fecal organic acids and rise in $\mathrm{pH}$ level elicited by surgical procedures.

Protection of the host intestines from exogenous pathogens by commensal bacteria is termed colonization resistance. Disruption of the barrier function (e.g. induced by antibiotic treatment) is associated with an increased risk of CD infection and/or flourish of multiresistant bacteria. Various possible mechanisms have been proposed to 
explain how the indigenous microbiome constitutes colonization resistance, including direct interactions between microbes as well as indirect mechanisms mediated by stimulation of the mucosal immune system by members of the health-associated microbiota [4,28-30]. An interesting aspect of our study is that positive correlations were observed among administered probiotics, the number of Bifidobacterium species and the concentrations of short chain fatty acids, which are closely associated with decreases in potentially harmful species, including CD. This finding from human samples may help explain the mechanisms whereby probiotics reduce colonization of pathogenic organisms through enhanced colonization resistance in the gastrointestinal tract; it may also support the view, proposed by Fukuda et al, that Bifidobacteria can protect against enteropathogenic infection through production of acetate [31,32].

\section{References}

1. Correia MI, Liboredo JC, Consoli ML (2012) The role of probiotics in gastrointestinal surgery. Nutrition 28: 230-234.

2. Gerritsen J, Smidt H, Rijkers GT, de Vos WM (2011) Intestinal microbiota in human health and disease: the impact of probiotics. Genes Nutr 6: 209-240.

3. Jeppsson B, Mangell P, Thorlacius H (2011) Use of probiotics as prophylaxis for postoperative infections. Nutrients 3: 604-612.

4. Lawley TD, Walker AW (2013) Intestinal colonization resistance Immunology 138: 1-11.

5. Deriu E, Liu JZ, Pezeshki M, Edwards RA, Ochoa RJ, et al. (2013) Probiotic bacteria reduce salmonella typhimurium intestinal colonization by competing for iron. Cell Host Microbe 14: 26-37.

6. Goldenberg JZ, Ma SS, Saxton JD, Martzen MR, Vandvik PO, et al. (2013) Probiotics for the prevention of Clostridium difficile-associated diarrhea in adults and children. Cochrane Database Syst Rev 5: CD006095.

7. Sassone-Corsi M, Raffatellu M (2015) No vacancy: how beneficial microbes cooperate with immunity to provide colonization resistance to pathogens. J Immunol 194: 4081-4087.

8. Kanazawa H, Nagino M, Kamiya S, Komatsu S, Mayumi T, et al. (2005) Synbiotics reduce postoperative infectious complications: a randomized controlled trial in biliary cancer patients undergoing hepatectomy. Langenbecks Arch Surg 390: 104-113.

9. Rayes N, Seehofer D, Theruvath T, Mogl M, Langrehr JM, et al. (2007) Effect of enteral nutrition and synbiotics on bacterial infection rates after pylorus-preserving pancreatoduodenectomy: a randomized, double-blind trial. Ann Surg 246: 36-41.

10. Rayes N, Seehofer D, Theruvath T, Schiller RA, Langrehr JM, et al. (2005) Supply of pre- and probiotics reduces bacterial infection rates after liver transplantation--a randomized, double-blind trial. Am J Transplant 5: 125-130.

11. Sugawara G, Nagino M, Nishio H, Ebata T, Takagi K, et al. (2006) Perioperative synbiotic treatment to prevent postoperative infectious complications in biliary cancer surgery: a randomized controlled trial. Ann Surg 244: 706-714.

12. Anderson AD, McNaught CE, Jain PK, MacFie J (2004) Randomised clinical trial of synbiotic therapy in elective surgical patients. Gut 53: 241-245.

13. He D, Wang HY, Feng JY, Zhang MM, Zhou Y, et al. (2013) Use of pro-/ synbiotics as prophylaxis in patients undergoing colorectal resection for cancer: a meta-analysis of randomized controlled trials. Clin Res Hepatol Gastroenterol 37: 406-415.

14. Horvat M, Krebs B, Potrc S, Ivanecz A, Kompan L (2010) Preoperative synbiotic bowel conditioning for elective colorectal surgery. Wien Klin Wochenschr 122 Suppl 2: 26-30.

15. Liu Z, Qin H, Yang Z, Xia Y, Liu W, et al. (2011) Randomised clinical trial: the effects of perioperative probiotic treatment on barrier function and post-operative infectious complications in colorectal cancer surgery - a double-blind study. Aliment Pharmacol Ther 33: 50-63.

16. Liu ZH, Huang MJ, Zhang XW, Wang L, Huang NQ, et al. (2013) The effects of perioperative probiotic treatment on serum zonulin concentration and subsequent postoperative infectious complications after colorectal cancer surgery: a double-center and double-blind randomized clinical trial. Am J Clin Nutr 97: 117-126

17. McNaught CE, Woodcock NP, MacFie J, Mitchell CJ (2002) A prospective randomised study of the probiotic Lactobacillus plantarum $299 \mathrm{~V}$ on indices of gut barrier function in elective surgical patients. Gut 51: 827-831.

18. Peitsidou K, Karantanos T, Theodoropoulos GE (2012) Probiotics, prebiotics, synbiotics: is there enough evidence to support their use in colorectal cancer surgery?. Dig Surg 29: 426-438.

19. Reddy BS, Macfie J, Gatt M, Larsen CN, Jensen SS, et al. (2007) Randomized clinical trial of effect of synbiotics, neomycin and mechanical bowel preparation on intestinal barrier function in patients undergoing colectomy. Br J Surg 94: 546-554.

20. Sadahiro S, Suzuki T, Tanaka A, Okada K, Kamata H, et al. (2014) Comparison between oral antibiotics and probiotics as bowel preparation for elective colon cancer surgery to prevent infection: prospective randomized trial. Surgery 155: 493-503.

21. Zhang JW, Du P, Gao J, Yang BR, Fang WJ, et al. (2012) Preoperative probiotics decrease postoperative infectious complications of colorectal cancer. Am J Med Sci 343: 199-205.

22. Komatsu S, Sakamoto E, Norimizu S, Shingu Y, Asahara T, et al. (2016) Efficacy of perioperative synbiotics treatment for the prevention of surgical site infection after laparoscopic colorectal surgery: a randomized controlled trial. Surg Today 46: 479-490.

23. Mizuno T, Yokoyama Y, Nishio H, Ebata T, Sugawara G, et al. (2010) Intraoperative bacterial translocation detected by bacterium-specific ribosomal rna-targeted reverse-transcriptase polymerase chain reaction for the mesenteric lymph node strongly predicts postoperative infectious complications after major hepatectomy for biliary malignancies. Ann Surg 252: 1013-1019.

24. Nishigaki E, Abe T, Yokoyama Y, Fukaya M, Asahara T, et al. (2014) The detection of intraoperative bacterial translocation in the mesenteric lymph nodes is useful in predicting patients at high risk for postoperative infectious complications after esophagectomy. Ann Surg 259: 477-484.

25. Yokoyama Y, Nishigaki E, Abe T, Fukaya M, Asahara T, et al. (2014) Randomized clinical trial of the effect of perioperative synbiotics versus no synbiotics on bacterial translocation after oesophagectomy. Br J Surg 101: 189-199.

26. Kotzampassi K, Stavrou G, Damoraki G, Georgitsi M, Basdanis G, et al. (2015) A Four-Probiotics Regimen Reduces Postoperative Complications After Colorectal Surgery: A Randomized, Double-Blind, PlaceboControlled Study. World J Surg 39: 2776-2783.

27. Watanabe M, Murakami M, Nakao K, Asahara T, Nomoto K, et al. (2010) Randomized clinical trial of the influence of mechanical bowel preparation on faecal microflora in patients undergoing colonic cancer resection. Br J Surg 97: 1791-1797.

28. Britton RA, Young VB (2012) Interaction between the intestinal microbiota and host in Clostridium difficile colonization resistance. Trends Microbiol 20: 313-319.

29. Britton RA, Young VB2 (2014) Role of the intestinal microbiota in resistance to colonization by Clostridium difficile. Gastroenterology 146: 1547-1553.

30. Buffie CG, Pamer EG (2013) Microbiota-mediated colonization resistance against intestinal pathogens. Nat Rev Immunol 13: 790-801.

31. Fukuda S, Toh H, Hase K, Oshima K, Nakanishi Y, et al. (2011) Bifidobacteria can protect from enteropathogenic infection through production of acetate. Nature 469: 543-547.

32. Fukuda S, Toh H, Taylor TD, Ohno H, Hattori M (2012) Acetateproducing bifidobacteria protect the host from enteropathogenic infection via carbohydrate transporters. Gut Microbes 3: 449-454. 